\title{
Electronically Controllable Fully-Uncoupled Explicit Current-Mode Quadrature Oscillator Using VDTAs and Grounded Capacitors
}

\author{
Dinesh Prasad $^{1 *}$, Mayank Srivastava ${ }^{2}$, Data Ram Bhaskar ${ }^{1}$ \\ ${ }^{1}$ Department of Electronics and Communication Engineering, Faculty of Engineering and Technology, \\ Jamia Millia Islamia, New Delhi, India \\ ${ }^{2}$ Department of Electronics and Communication Engineering, Amity School of Engineering and Technology, \\ Amity University, Noida, India \\ Email: ${ }^{*}$ dprasad@jmi.ac.in, mayank2780@gmail.com, dbhaskar@jmi.ac.in
}

Received October 5, 2012; revised November 27, 2012; accepted December 5, 2012

Copyright (C) 2013 Dinesh Prasad et al. This is an open access article distributed under the Creative Commons Attribution License, which permits unrestricted use, distribution, and reproduction in any medium, provided the original work is properly cited.

\begin{abstract}
An electronically controllable fully uncoupled explicit current-mode quadrature oscillator employing Voltage Differencing Transconductance Amplifiers (VDTAs) as active elements has been presented. The proposed configuration employs two VDTAs along with grounded capacitors and offers the following advantageous features 1) fully and electronically independent control of condition of oscillation (CO) and frequency of oscillation (FO); 2) explicit currentmode quadrature oscillations; and 3) low active and passive sensitivities. The workability of proposed configuration has been demonstrated by PSPICE simulations with TSMC CMOS $0.18 \mu \mathrm{m}$ process parameters.
\end{abstract}

Keywords: VDTA; Quadrature Oscillator; Current-Mode Circuits

\section{Introduction}

Among various kinds of oscillators, the quadrature oscillators (QO) are widely used because they can provide two sinusoids with $\pi / 2$ phase difference, for example, in telecommunication systems, for quadrature mixtures and single-sideband generators or for measurement purposes in vector generators or selective voltmeters $[1,2]$. Therefore the QO play an important role in many communication systems, instrumentation systems and signal processing see [3-11]. Recently, a CMOS realization of VDTA and its applications as 1) RF filter and 2) double tuned amplifier have been presented in [12]. In [13], an electronically controllable explicit current-output sinusoidal oscillator has been reported. Another application as a single input five output voltage-mode universal filter using VDTAs has been presented in [14]. The purpose of this communication is to introduce a new electronically controllable fully uncoupled explicit current-mode quadrature oscillator using two VDTAs and two grounded capacitors. The proposed configuration provides the advantageous features of: 1) completely and electronically independent control of condition of oscillation (CO) and

${ }^{*}$ Corresponding author. frequency of oscillation (FO); 2) explicit current-mode quadrature oscillations; and 3) low active and passive sensitivities. The workability of proposed configuration has been verified using SPICE simulation with TSMC CMOS $0.18 \mu \mathrm{m}$ process parameters.

\section{The Proposed Configurations}

The symbolic notation of the VDTA is shown in Figure 1, where $V_{P}$ and $V_{N}$ are input terminals and $Z, X^{+}$and $X$ are output terminals. All terminals of VDTA exhibit high impedance values [12]. The VDTA can be described by the following set of equations:

$$
\begin{aligned}
& {\left[\begin{array}{c}
I_{Z} \\
I_{X^{+}} \\
I_{X^{-}}
\end{array}\right]=\left[\begin{array}{ccc}
g_{m_{1}} & -g_{m_{1}} & 0 \\
0 & 0 & g_{m_{2}} \\
0 & 0 & -g_{m_{2}}
\end{array}\right]\left[\begin{array}{c}
V_{V_{P}} \\
V_{V_{N}} \\
V_{Z}
\end{array}\right]}
\end{aligned}
$$

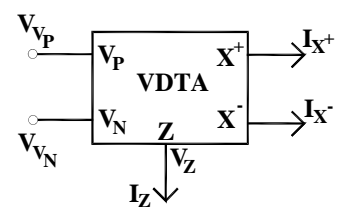

Figure 1. The symbolic notation of VDTA. 
The proposed configuration is shown in Figure 2.

Circuit analysis of Figure 2 gives the characteristic equation $(\mathrm{CE})$ as:

$$
s^{2}+s \frac{1}{C_{2}}\left(g_{m_{4}}-g_{m_{3}}\right)+\frac{g_{m_{1}} g_{m_{2}}}{C_{1} C_{2}}=0
$$

From Equation (2), the $\mathrm{CO}$ and $\mathrm{FO}$ can be expressed as $\mathrm{CO}$ :

$$
\left(g_{m_{4}}-g_{m_{3}}\right) \leq 0
$$

and

FO:

$$
\omega_{0}=\sqrt{\frac{g_{m_{1}} g_{m_{2}}}{C_{1} C_{2}}}
$$

Therefore, it is seen from Equations (3) and (4) that the $\mathrm{CO}$ and FO are completely uncoupled and electronically tunable as $g_{m_{i}} ; i=1-4$ are controlled by bias currents.

The current transfer functions obtained from Figure 2 are given by:

$$
\frac{I_{o 2}(s)}{I_{o 1}(s)}=\frac{g_{m_{4}}}{g_{m_{1}} g_{m_{2}} s C_{1}}
$$

For sinusoidal steady state, Equations (5) becomes

$$
\frac{I_{o 2}(j \omega)}{I_{o 1}(j \omega)}=\frac{g_{m_{4}}}{\omega C_{1} g_{m_{1}} g_{m_{2}}} \mathrm{e}^{-j 90^{\circ}}
$$

Thus, the phase difference $\phi$ between $\left(I_{o 2}\right.$ and $\left.I_{o 1}\right)$ is $-90^{\circ}$. Hence, the currents $\left(I_{o 2}\right.$ and $\left.I_{o 1}\right)$ are in the quadrature form.

\section{Parasitic Effects and Sensitivity Analysis}

By considering the various VDTA non-ideal parameters like the finite $P$-terminal parasitic impedance consisting of a resistance $R_{P}$ in parallel with capacitance $C_{P}$, the finite $N$-terminal parasitic impedance consisting of a resistance $R_{N}$ in parallel with capacitance $C_{N}$, the finite $X$-terminal parasitic impedance consisting of a resistance $R_{X}$ in parallel with capacitance $C_{X}$ and the parasitic impedance at the $Z$-terminal consisting of a resistance $R_{Z}$ in parallel with capacitance $C_{Z}$ then the expression of $\mathrm{CO}$ and $\mathrm{FO}$ including the influence of parasitic are given by:

$\mathrm{CO}$ :

$$
\begin{aligned}
& \left\{\frac{2 C_{1}}{R_{p}}+\frac{C_{1}}{R_{z}}+\frac{2 C_{1}}{R_{x}}+\frac{2 C_{p}}{R_{z}}+\frac{C_{2}}{R_{z}}+\frac{C_{z}}{R_{z}}+\frac{2 C_{x}}{R_{z}}+\frac{2 C_{z}}{R_{p}}\right. \\
& \left.+\frac{C_{z}}{R_{z}}+\frac{2 C_{z}}{R_{x}}+C_{1}\left(g_{m_{4}}-g_{m_{3}}\right)+C_{z}\left(g_{m_{4}}-g_{m_{3}}\right)\right\} \leq 0
\end{aligned}
$$

then the active and passive sensitivities of $\omega_{0}$ can be found as:

$$
\begin{gathered}
S_{R_{x}}^{\omega_{0}}=\frac{-1}{R_{x} R_{z}\left(\frac{2}{R_{p} R_{z}}+\frac{1}{R_{z}^{2}}+\frac{2}{R_{x} R_{z}}+\frac{g_{m_{4}}}{R_{z}}-\frac{g_{m_{3}}}{R_{z}}+g_{m_{1}} g_{m_{2}}\right)}, S_{R_{z}}^{\omega_{0}}=\frac{\left(-\frac{2}{R_{p} R_{z}}-\frac{2}{R_{z}^{2}}-\frac{2}{R_{x} R_{z}}-\frac{g_{m_{4}}}{R_{z}}+\frac{g_{m_{3}}}{R_{z}}\right)}{2\left(\frac{2}{R_{p} R_{z}}+\frac{1}{R_{z}{ }^{2}}+\frac{2}{R_{x} R_{z}}+\frac{g_{m_{4}}}{R_{z}}-\frac{g_{m_{3}}}{R_{z}}+g_{m_{1}} g_{m_{2}}\right)}, \\
S_{R_{P}}^{\omega_{0}}=\frac{-1}{R_{P} R_{z}\left(\frac{2}{R_{p} R_{z}}+\frac{1}{R_{z}^{2}}+\frac{2}{R_{x} R_{z}}+\frac{g_{m_{4}}}{R_{z}}-\frac{g_{m_{3}}}{R_{z}}+g_{m_{1}} g_{m_{2}}\right)}, S_{g_{m_{1}}}^{\omega_{0}}=\frac{g_{m_{1}} g_{m_{2}}}{2\left(\frac{2}{R_{p} R_{z}}+\frac{1}{R_{z}^{2}}+\frac{2}{R_{x} R_{z}}+\frac{g_{m_{4}}}{R_{z}}-\frac{g_{m_{3}}}{R_{z}}+g_{m_{1}} g_{m_{2}}\right)}=S_{g_{m_{2}}}^{\omega_{0}}, \\
S_{g_{m_{3}}}^{\omega_{0}}=\frac{-g_{m_{4}}}{2 R_{z}^{2}\left(\frac{2}{R_{p} R_{z}}+\frac{1}{R_{z}^{2}}+\frac{2}{R_{x} R_{z}}+\frac{g_{m_{4}}}{R_{z}}-\frac{g_{m_{3}}}{R_{z}}+g_{m_{1}} g_{m_{2}}\right)}, S_{g_{m_{4}}}^{\omega_{0}}=\frac{g_{m_{4}}}{2 R_{z}^{2}\left(\frac{2}{R_{p} R_{z}}+\frac{1}{R_{z}^{2}}+\frac{2}{R_{x} R_{z}}+\frac{g_{m_{1}} g_{m_{2}}}{R_{z}}\right),} \\
S_{C_{1}}^{\omega_{0}}=\frac{-C_{2}\left(C_{2}+2 C_{p}+C_{z}+2 C_{x}\right)}{2\left(C_{1} C_{2}+2 C_{1} C_{p}+C_{1} C_{z}+2 C_{1} C_{x}+2 C_{p} C_{z}+C_{2} C_{z}+C_{z}^{2}+2 C_{x} C_{z}\right)}, \\
-C_{2}\left(C_{1}+C_{z}\right)
\end{gathered}
$$




$$
\begin{aligned}
& S_{C_{x}}^{\omega_{0}}=\frac{-2 C_{x}\left(C_{1}+C_{z}\right)}{2\left(C_{1} C_{2}+2 C_{1} C_{p}+C_{1} C_{z}+2 C_{1} C_{x}+2 C_{p} C_{z}+C_{2} C_{z}+C_{z}^{2}+2 C_{x} C_{z}\right)}, \\
& S_{C_{P}}^{\omega_{0}}=\frac{-2 C_{P}\left(C_{1}+C_{z}\right)}{2\left(C_{1} C_{2}+2 C_{1} C_{p}+C_{1} C_{z}+2 C_{1} C_{x}+2 C_{p} C_{z}+C_{2} C_{z}+C_{z}^{2}+2 C_{x} C_{z}\right)}, \\
& S_{C_{z}}^{\omega_{0}}=\frac{-C_{z}\left(C_{1}+2 C_{p}+C_{2}+2 C_{z}+2 C_{x}\right)}{2\left(C_{1} C_{2}+2 C_{1} C_{p}+C_{1} C_{z}+2 C_{1} C_{x}+2 C_{p} C_{z}+C_{2} C_{z}+C_{z}^{2}+2 C_{x} C_{z}\right)}
\end{aligned}
$$

For $C_{1}=C_{2}=0.5 \mathrm{nF}, R_{p}=R_{z}=\infty, C_{p}=C_{x}=C_{z}=0.15$ $\mathrm{pF}, g_{m_{1}}=g_{m_{2}}=g_{m_{3}}=1.5913 \mathrm{~mA} / \mathrm{V}$ and $g_{m_{4}}=$ $1.7916 \mathrm{~mA} / \mathrm{V}$, the sensitivities are found to be $0,0,0,0.5$, $0,0,-0.899,-0.499,-2.9 \mathrm{e}-4,-2.9 \mathrm{e}-4,-1.64 \mathrm{e}-3$ for Equation (9). Thus, all the active and passive sensitivities of $\omega_{0}$ with respect to each active and passive elements are low.

\section{Simulation Results}

To verify the theoretical analysis, the proposed ECMSO was simulated using CMOS VDTA from [12]. Power supply voltages were taken as $V_{D D}=-V_{S S}=0.9 \mathrm{~V}$ and $I_{B 1}$ $=I_{B 2}=I_{B 3}=I_{B 4}=2 \mathrm{~mA}$ (for $\mathrm{VDTA}_{1}$ ) and $I_{B 1}=I_{B 2}=2$ $\mathrm{mA}, I_{B 3}=I_{B 4}=3.687 \mathrm{~mA}$ (for $\mathrm{VDTA}_{2}$ ) biasing currents are used. The transistor aspect ratios are same as in [12]. The passive elements of the configuration were selected as $C_{1}=C_{2}=0.5 \mathrm{nF}$. The transconductances of VDTA were controlled by bias currents. PSPICE generated output waveforms indicating transient and steady state responses are shown in Figures 3(a) and (b) respectively. These results, thus, confirm the validity of the proposed configuration. The total harmonic distortion (THD) of the proposed oscillator is found to be $3.00 \%$ (Figure 4). From Figure 5 it is clear that the two currents are in quadrature.

Figure 5 shows that the two currents are in quadrature and the measured value of phase shift between two waveforms is $=-89.98^{\circ}$.

\section{Concluding Remarks}

In this paper, an explicit current-mode quadrature oscillator using VDTAs has been presented. The presented circuit employs two VDTAs and two grounded capaci-

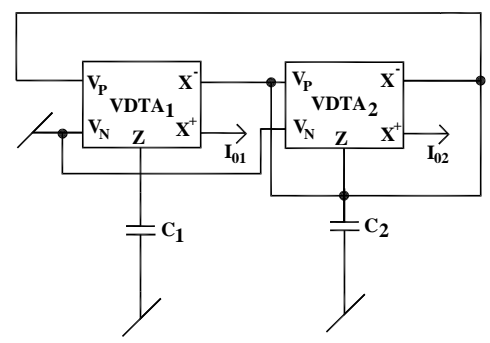

Figure 2. The proposed configuration.

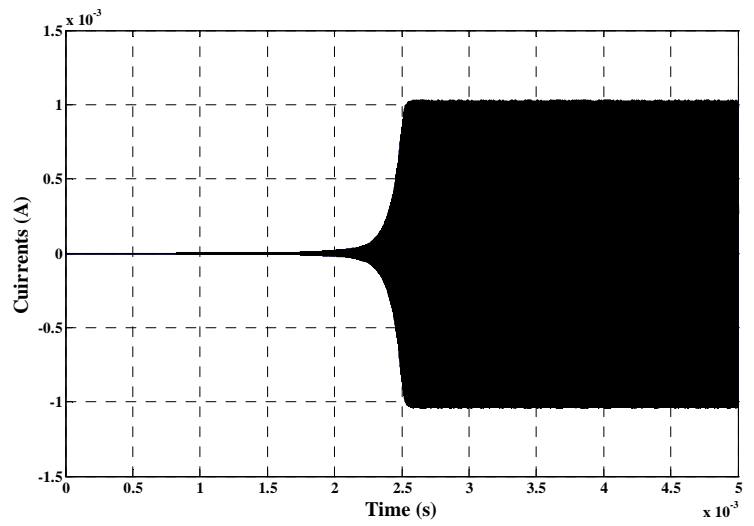

(a)

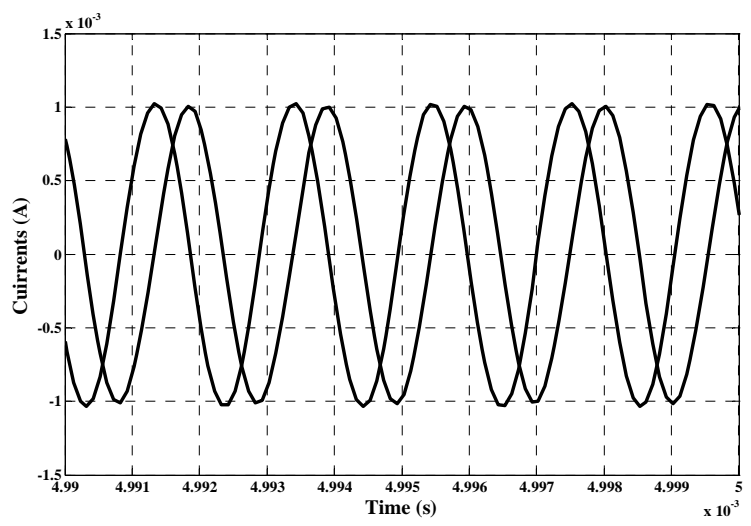

(b)

Figure 3. (a) Transient response waveform; (b) Steady state response of the quadrature outputs.

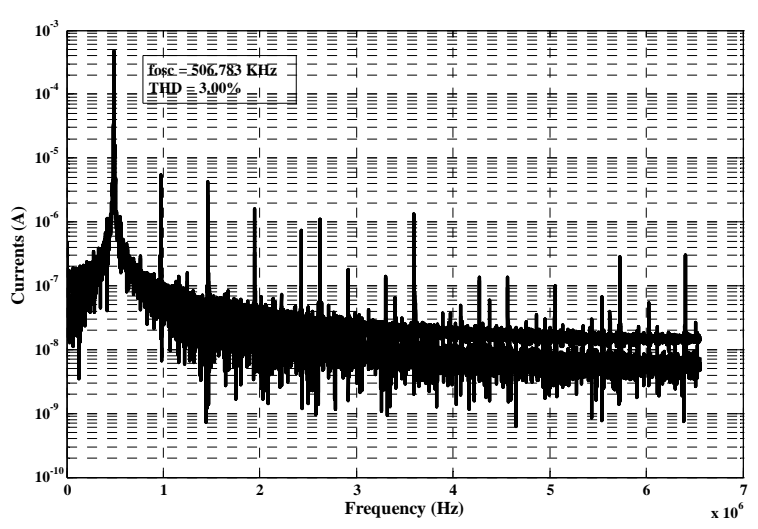

Figure 4. Simulation result of the output spectrum. 


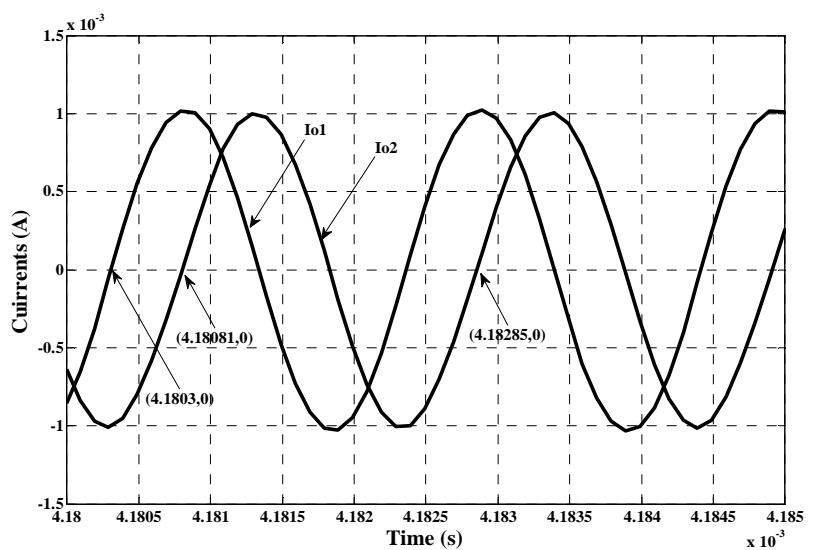

Figure 5. Steady state response of the quadrature outputs of $I_{o 2}$ and $I_{o 1}$.

tors. The $\mathrm{CO}$ and $\mathrm{FO}$ of the proposed quadrature oscillator has the advantage of fully and electronically independent controllability. The proposed explict currentmode quadrature oscillator also provides low active and passive sensitivities. The workability of proposed configuration has been verified using SPICE simulation.

\section{REFERENCES}

[1] J. W. Horng, "Current Conveyors Based Allpass Filters and Quadrature Oscillators Employing Grounded Capacitors and Resistors," Computer and Electrical Engineering, Vol. 31, No. 1, 2005, pp. 81-92. doi:10.1016/j.compeleceng.2004.11.006

[2] D. Prasad, D. R. Bhaskar and A. K. Singh, "Electronically Controllable Grounded Capacitor Current-Mode Quadrature Oscillator Using Single MO-CCCDTA," Radioengineering, Vol. 20, No. 1, 2011, pp. 354-359.

[3] W. Tangsrirat and W. Tanjaroen, "Current-Mode Sinusoidal Oscillator with Independent Control of Oscillation Frequency and Condition Using CDTAs," Indian Journal of Pure and Applied Physics, Vol. 48, No. 5, 2010, pp. 363-366.

[4] M. T. Abuelmatti, "New OTA-Based Sinusoidal Oscillators with Fully Uncoupled Control of Oscillation Frequency and Condition," Frequenz: Journal of RF-Engineering and Telecommunications, Vol. 55, No. 7-8, 2001, pp. 224-228.

[5] M. T. Abuelmatti, "Active-Only Sinusoidal Oscillator with Electronically-Tunable Fully-Uncoupled Frequency and Condition of Oscillation," Active and Passive Electronic Components, Vol. 24, No. 4, 2001, pp. 233-241. doi:10.1155/2001/27076

[6] M. T. Abuelmatti and H. A. Al-Zaher, "Current Mode Quadrature Sinusoidal Oscillators Using Two FTFNs," Frequenz: Journal of RF-Engineering and Telecommunications, Vol. 53, No. 1-2, 1999, pp. 27-30.

[7] W. Tangsrirat and S. Pisitchalermpong, "CDBA-Based Quadrature Sinusoidal Oscillator," Frequenz: Journal of RF-Engineering and Telecommunication, Vol. 61, No. 34, 2007, pp. 102-104.

[8] W. Tangsrirat, D. Prasertsoma, T. Piyatata and W. Surakampontorn, "Single-Resistance-Controlled Quadrature Oscillator Using Current Differencing Buffered Amplifiers," International Journal of Electronics, Vol. 95, No. 11, 2008, pp. 1119-1126. doi:10.1080/00207210802387676

[9] M. Kumngern and K. Dejhan, "DDCC-Based Quadrature Oscillator with Grounded Capacitors and Resistors," Journal of Active and Passive Electronic Component, 2009, Article ID: 987304.

[10] A. Lahiri, "Current Mode Variable Frequency Quadrature Oscillator Using Two CCs and Four Passive Component Including Grounded Capacitors," Analog Integrated Circuits and Signal Processing, Vol. 68, No. 1, 2011, pp. 129-131. doi:10.1007/s10470-011-9635-4

[11] M. T. Abuelmatti, "New Sinusoidal Oscillators with Fully Uncoupled Control of Oscillation Frequency and Condition Using Three CCII+s," Analog Integrated Circuits and Signal Processing, Vol. 24, No. 3, 2000, pp. 253-261.

[12] A. Yesil, F. Kacar and H. Kuntman, "New Simple CMOS Realization of Voltage Differencing Transconductance Amplifier and Its RF Filter Application," Radioengineering, Vol. 20, No. 3, 2011, pp. 632-637.

[13] D. Prasad and D. R. Bhaskar, "Electronically-Controllable Explicit Current Output Sinusoidal Oscillator Employing Single VDTA," ISRN Electronics, Vol. 2012, 2012, Article ID: 382560 . doi: $10.5402 / 2012 / 382560$

[14] J. Satansup, T. Pukkalanun and W. Tangsrirat, "Electronically Tunable Single-Input Five-Output Voltage-Mode Universal Filter Using VDTAs and Grounded Passive Elements," Circuits, Systems and Signal Processing, 2012. doi:10.1007/s00034-012-9492-0 